Recent Advances in Communication, Electronics \& Electrical Engineering

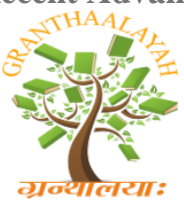

INTERNATIONAL JOURNAL OF RESEARCH -

GRANTHAALAYAH

A knowledge Repository

RACEEE - 17

\title{
PD CONTROLLER BASED UNMANNED SHIP NAVIGATION
}

\author{
Adinath Jain ${ }^{* 1}$, Dr. S.A. Hari Prasad ${ }^{2}$, Sujata Dahal ${ }^{3}$, Rajesh Sudi ${ }^{4}$ \\ ${ }^{*}$ Assistant Professor EEE, R V College of Engineering, India \\ ${ }^{2}$ Director, School of Engineering and Technology, Jain University, India \\ ${ }^{3}$ Embedded System Design, School of Engineering and Technology, Jain University, India \\ ${ }^{4}$ Proprietor, BRAINS, India
}

DOI: https://doi.org/10.29121/granthaalayah.v5.i4RACEEE.2017.3337

\begin{abstract}
For the improvement of the performance of track keeping of unmanned water vehicle numerous ship models, wave disturbances models and distinct control algorithms has been proposed. Researchers are using variants of PID controllers, Adaptive controllers and Predictive controllers for accurate trajectory control. Being simpler in nature still PID controller are popular in control domain. In this paper we have implemented PD based controller for trajectory control of unmanned vehicle considering all standard models of sea disturbances. Analysis of result obtained using PD control proved that path tracking is more accurate than open loop controller in terms of computation time, complexity and fuel consumption.
\end{abstract}

Keywords: PD Controller; Tracking Efficiency.

Cite This Article: Adinath Jain, Dr. S.A. Hari Prasad, Sujata Dahal, and Rajesh Sudi. (2017). "PD CONTROLLER BASED UNMANNED SHIP NAVIGATION." International Journal of Research - Granthaalayah, 5(4) RACEEE, 147-154.

\section{Introduction}

In ship navigation, accurate ship tracking is very crucial as it saves time and fuel consumption. In a track keeping, the autopilot takes the ship's position information and uses it to calculate heading corrections to make ship follows a predetermined course. The current position and the waypoints of the ship determine the desired heading angle. The desired heading angle is time varying as it changes with ship's current position and time. Reliability, cost effectiveness and safety of the used control system are also another matter of concern. For this, a robust controller capable of generating accurate heading angle considering sea disturbances, ship hydrodynamics and noises (both internal and external) is required. [1] Mathematical model can be divided into classical and analytical modeling. Analytical model is based on physical knowledge of the system whereas mathematical model is an experimental approach where experiments are performed on the system and model is then fitted to the record data. Nomoto model, Norrbin 
Recent Advances in Communication, Electronics \& Electrical Engineering model, Bech model etc are examples of classical ship modeling techniques. The Nomoto Model is one of the simple linear models, supporting 6 DOF that are used for modeling of ship dynamics. Bretschnieder spectrum, Pierson-Markowitz (P-M) spectrum, Modified PiersonMarkowitz and JONSWAP spectrum are several techniques used for wave modeling. Among them P-M spectrum supports fully developed sea elevation and considers wide range of wave frequencies considering all possible wind speeds.

Different controllers like PID, Predictive and adaptive controller are used for ship tracking. Due to parameters like environmental changes, random disturbances, internal errors etc. controller should be able to change automatically. Various algorithms like genetic algorithm, fuzzy logic can be implemented along with PD controller to automatically accommodate controller coefficients $K_{p}, K_{d}$. Adaptive controller adjust its gain parameters in accordance with environment for optimum solutions but it cannot predict future control output of the ship. Predictive controller can predict future outputs and can tune control parameter for future steps but it is computationally intensive in nature.

In 1960s Proportional Integral Derivative (PID) control, in 1970s Multiple input Multiple output (MIMO) control with Kalman filter and nonlinear control from 1990s to present including feedback linearization, adaptive control, fuzzy control, backstepping, Sliding Mode Control (SMC), neural network control, Model predictive control (MPC) and hybrid control have been extensively used for tracking control of surface vessels. [2] Dr. S. A. Hariprasad et al. compares the performance of PID and Predictive controllers to evaluate tracking efficiency and computational time. Further this paper concludes that being computationally intensive also predictive controller is well suited for better tracking. [5] Ke Feng et al, proposed an adaptive fuzzy logic control scheme with backstepping scheme, based on uncertain strict feedback nonlinear system with unknown dynamics and unpredictable disturbances, to track unmanned surface vehicles [6] To trace the trajectory and the altitude of the unmanned underwater vehicle with unpredictable disturbances and thruster dynamics, Yan-Cheng Lie et al. proposed a Fully Tuned Fuzzy Neural Network Based Robust Adaptive Control (FFNNBARC). A back-stepping control law is approximated by the FFNN estimator. Similarly, the finite $\mathrm{L}_{2}$ gain property provided by the designed robust controller reconstructs errors and makes overall control system robust. [7] To track an unmanned surface vehicle being suffered from an unknown dynamics and external disturbances Ning Wang et al, proposed an Extreme Learning Control (ELC) framework with random hidden node. To cover unknown and unpredictable dynamics and external hindrances an error surface and transformed states are defined. Similarly, to precisely identify lumped non-linearity without having prior knowledge of a system or without tuning weights, the Single Feed-forward network is used.[8] Chang-Zhong et al. proposed an algorithm based on Lyapunov stability analysis to guarantee the error signals to be Uniformly Ultimately Bounded (UUB). This makes autonomous vehicle to follow the desired path more accurately via online learning neural networks. [9] The combination of adaptive switching based on nonlinear Lyapunov tracking control law is used to overcome convergence problem of position tracking error of the fully actuated autonomous vehicle. Since the vehicle route is independent of the path shape this is smoothen bounded curve parameterized by time. [10]. KhacDuc Do designed robust adaptive output feedback controller based on constructive method and Lyapunov's direct method for dynamic positioning of unmanned surface vehicle with unknown ship parameters under unpredictable disturbances as sea waves, water currents and wind. An adaptive observer is used 
Recent Advances in Communication, Electronics \& Electrical Engineering to estimate ship's velocities and position. This observer is further used to reduce high frequency noise. [11] Yuanhui Wang et al. used Pierson-Moskowitz (P-M) standard wave equation to simulate a random long-crested sea wave. Using mathematical wave disturbance model random wave disturbance force and moment acting on a large ship is calculated and considering sea state 4 real time simulations is done. Observed simulation results on the track and course change of large ship showed that the ocean wave had great influence on 4 degree of freedom (DOF) large ship which cannot be ignored. This paper is organized as follows: Section 2 describes the Nomoto ship model. Section 3 presents sea disturbances and Pierson-Moskowitz spectrum for modeling sea wave disturbances. The detail of proposed block diagram is given in section 4 along with the algorithm used. Simulation studies elucidating the performance of the proposed controller are presented in section 5 and Section 6 presents concluding remarks of the used controllers.

\section{Ship Model}

The ship is a rigid body with six degree of freedom. [3] The dynamics of ship is simulated by using a nonlinear model representing all relevant characteristics like hydrodynamic force, rudder and propeller thrust, and even effect of normal and low speed, shallow water, drift angle etc. But this model is too complicated and is not wise option for unmanned ship. So, the linear model is preferred over nonlinear

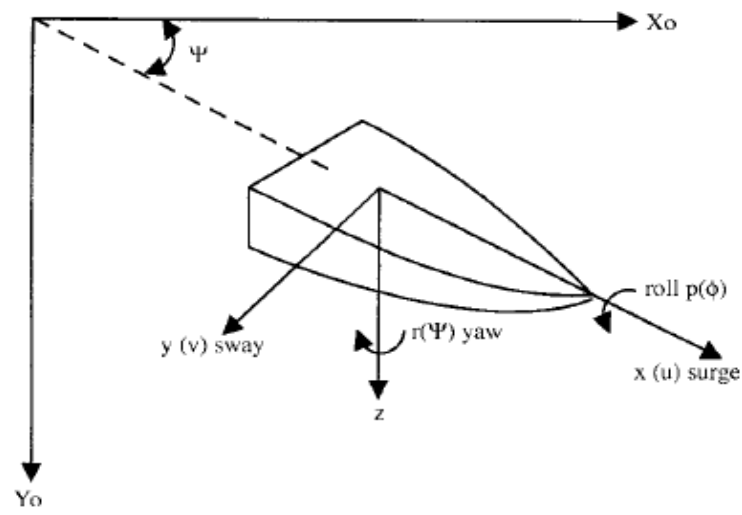

Figure 1: Sway-yaw-roll motion coordinate system

Among many ship modeling techniques, Nomoto model is simple, linear and most widely used. It supports all $6 \mathrm{DOF}$ and provides better performance for smaller rudder angle. Considering a constant forward speed $u_{0}$, decoupling linear surge equation, following transfer function is obtained. Further sway speed V and roll angle $\varphi$ are also eliminated.

$$
\frac{r}{\delta}=\frac{k\left(1+T_{3} S\right)\left(S^{2}+2 \eta \omega_{0} S+\omega_{0}^{2}\right)}{\left(1+T_{1} S\right)\left(1+T_{2} S\right)\left(S^{2}+2 \xi \omega_{n} S+\omega_{n}^{2}\right)}
$$

Due to coupling effect of roll on the yaw rate there are quadratic factors causing overshoot. Coupling effect from the sway mode to the yaw dynamics are due to the zero $\left(1+\mathrm{T}_{3} \mathrm{~S}\right)$ and the pole $\left(1+T_{2} S\right)$. If the roll mode is neglected, above transfer function can be further deducted to the following form, i.e. the second order transfer function. 


$$
\frac{\varphi}{\delta}=\frac{k\left(1+T_{3} S\right)}{\left(1+T_{1} S\right)\left(1+T_{2} S\right)}
$$

Here, $\mathrm{K}$ is static yaw rate gain and $\mathrm{T}_{1}, \mathrm{~T}_{2}$ and $\mathrm{T}_{3}$ are time constants.

\section{Modeling of Sea Disturbances}

Various environmental phenomenons like wind, waves and water current are responsible for undesirable motion of a ship. [4] Among them sea waves are more dominant. Initially small wavelets appearing on the water surface accounts the drag force allowing short waves to rise till their final break, producing energy. For example, a developing sea and storm with high frequencies creates a spectrum with relatively high peak. A storm blowing for a long time results a fully developed sea. Since sea waves are random in nature, the dynamics of sea states are taken into account to predict the ship movements. Wave's nature varies depending upon its source of origin but in this paper only wave generated from the wind is considered. Wave's pattern is the superposition of waves with different amplitudes, frequencies, phases and direction of propagations. But for the simplicity the stochastic approach considering frequency is taken in account and final spectrum is derived by applying Fast Fourier Transformation (FFT) on the measured data of wave motions.

$$
S(\omega)=C \omega^{-6} \exp \left(-2 g^{2} \omega^{-2} V^{-2}\right) \quad \mathrm{m}^{2} \mathrm{~s}
$$

Where, $\mathrm{C}$ is empirical constant $\mathrm{V}$ is the wind speed and $\mathrm{g}$ is acceleration due to gravity. Similarly, the earlier work of Phillips showed that the high frequency form of the sea wave is calculated as,

$$
S(\omega)=\alpha g^{2} \omega^{-5} \quad \mathrm{~m}^{2} \mathrm{~s}
$$

where, $\alpha$ is a positive constant. In this paper we have used Pierson-Moskowitz spectrum. This is a wave spectral formulation for fully developed wind-generated seas developed by the analyses of wave spectrum in the North Atlantic Ocean. The Pierson-Moskowitz spectrum is written as,

$$
S(\omega)=A \omega^{-5} \exp \left(-B \omega^{-4}\right) \quad \mathrm{m}^{2} \mathrm{~s}
$$

Where, $\mathrm{A}=8.1 * 10^{-3} g^{2}$, and $\mathrm{B}=0.74 * \frac{g}{v}^{4}$

Here, ' $\mathrm{V}$ ' is the wind speed at a height of $19.4 \mathrm{~m}$ over the sea surface, and ' $\mathrm{g}$ ' is the gravitational constant $\left(9.8 \mathrm{~m} / \mathrm{s}^{2}\right)$. It is assumed that the waves are represented by Gaussian random process and that $S(\omega)$ is narrow banded. Now, the concept of significant wave height is used to reformulate the PM-spectrum as:

$\mathrm{A}=8.1 * 10^{-3} \mathrm{~g}^{2}$ and $\mathrm{B}=0.0323 *{\frac{\mathrm{g}}{\mathrm{H}_{\mathrm{s}}^{2}}}^{2}=\frac{3.11}{H_{S}^{2}}$, and the maximum value of $S(\omega)$ is, 
Recent Advances in Communication, Electronics \& Electrical Engineering

$$
S_{\max }(\omega)=S\left(\omega_{0}\right)=\frac{5 A * \exp \left(-\frac{5}{4}\right)}{4 B \omega_{0}}
$$

where,

$$
\omega_{0}=\sqrt[4]{\frac{4 B}{5}}
$$

\section{Proposed System}

The proposed system aims at the trajectory control of an unmanned surface water vehicle. The block diagram consists of guidance system for track keeping, controller to follow the path given by guidance system and feedback loop for error minimization and for accurate performance. Proportional Derivative (PD) controller is used and its output behaviors are analyzed.

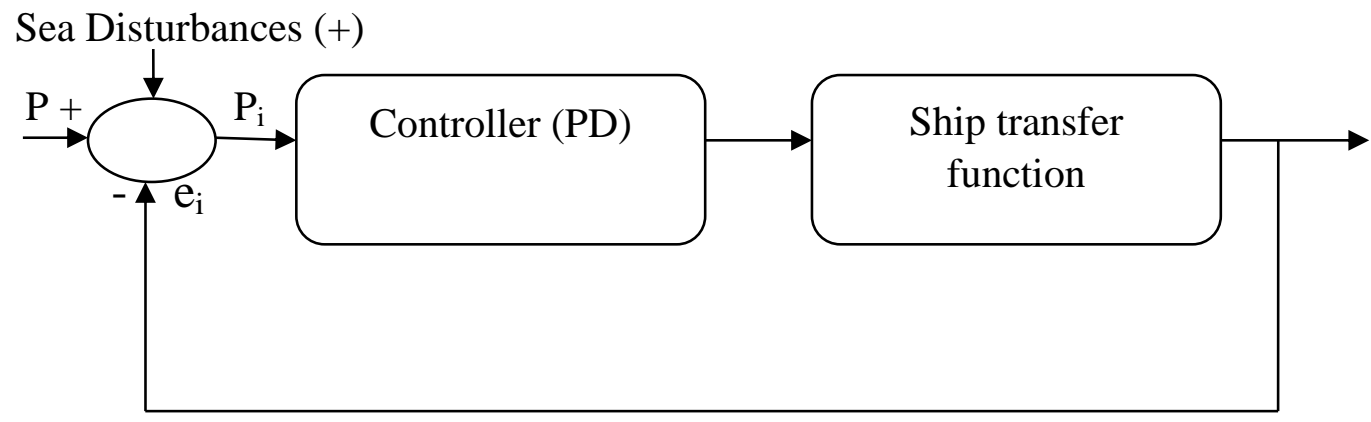

Figure 2: Block Diagram of PD based unmanned surface

\section{Simulation Results}

In this section, numerical simulations are performed for the comparison of the effectiveness of the controllers. The following parameters of the Nomoto model is used, $\mathrm{T}_{1}=10, \mathrm{~T}_{2}=20, \mathrm{~T}_{3}=40, \omega_{0}=3.2$ and $\omega_{n}=5, \mathrm{~K}=0.185, \eta=1$

So, transfer function for fourth order reduces to,

$$
\frac{r}{\delta}=\frac{7.4 s^{3}+47.54 s^{2}+76.96 s+1.8944}{200 s^{4}+2030 s^{3}+2349 s^{2}+760 s+25}
$$

Similarly, for second order, transfer function is:

$$
\frac{\psi}{\delta}=\frac{40 s+1}{200 s^{2}+30 s+1}
$$

In both fourth order and second order transfer functions, since all poles and zeros lie on the lefthalf of the plane, the system is stable according to Routh-Hurwitz stability criteria. Though plotting of transfer function characteristics without PD controller shows settling time of 100 
Recent Advances in Communication, Electronics \& Electrical Engineering seconds, plotting of poles and zeros, bode plot, and root-locus showed that the given system is stable.
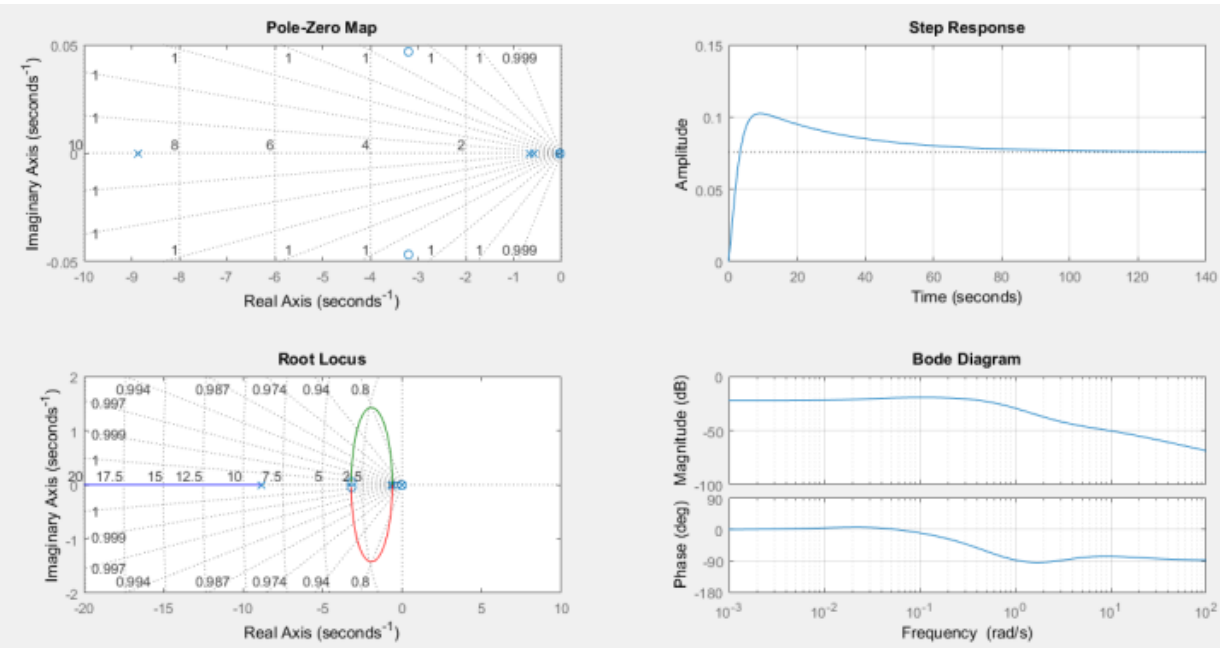

Figure 3a: Stability plot of 4th order TF
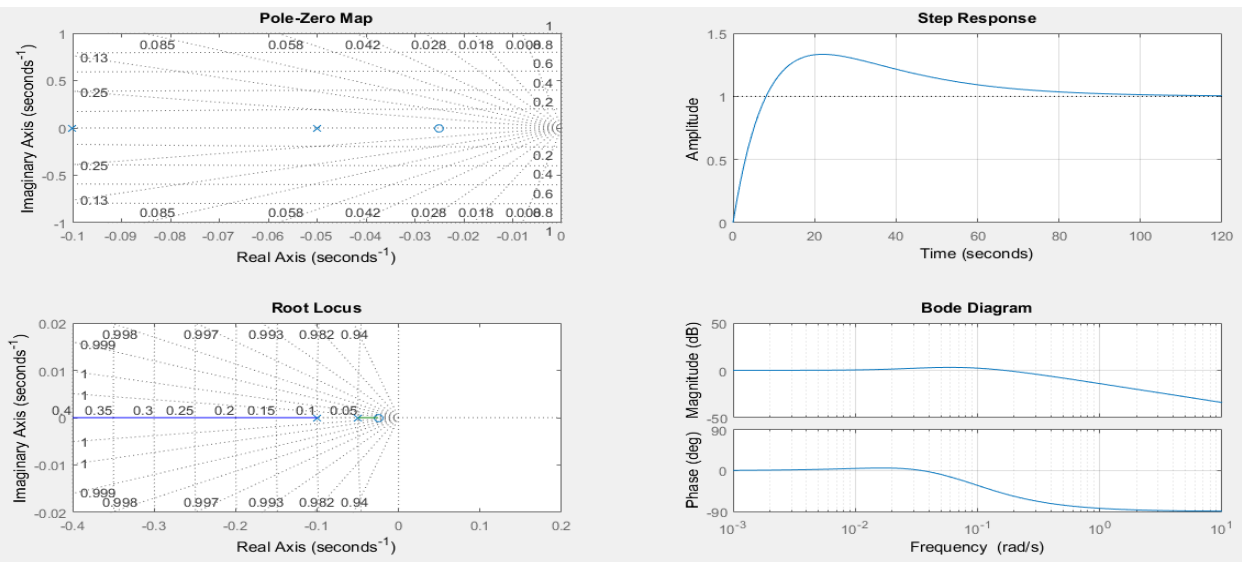

Figure 3b: Stability of 2nd order TF

Sea condition is assumed to be high and very high with the wave height ranging from 6.0 to 14.0 and the velocity of wind is assumed to be $19.3 \mathrm{~m} / \mathrm{s}^{2}$.

PD controller with the gain values P: 9087.088 and D: 0 are taken for fourth order ship's transfer function and P: 9090.9090 and D: 0 are taken for second order ship's transfer function. While tuning, settling time of 0.015 seconds is taken whereas in output settling time is $0.00 \mathrm{sec}$ while considering fourth order transfer function. Similarly, input settling time of 0.003 second is taken and output settling angle is found as 0.00 seconds in case of second order ship transfer functions too. The output track almost follows input track with no settling time at all. But without PD, in both cases it can be seen that not only output track is not following input but the desired waypoints are also not reached. 


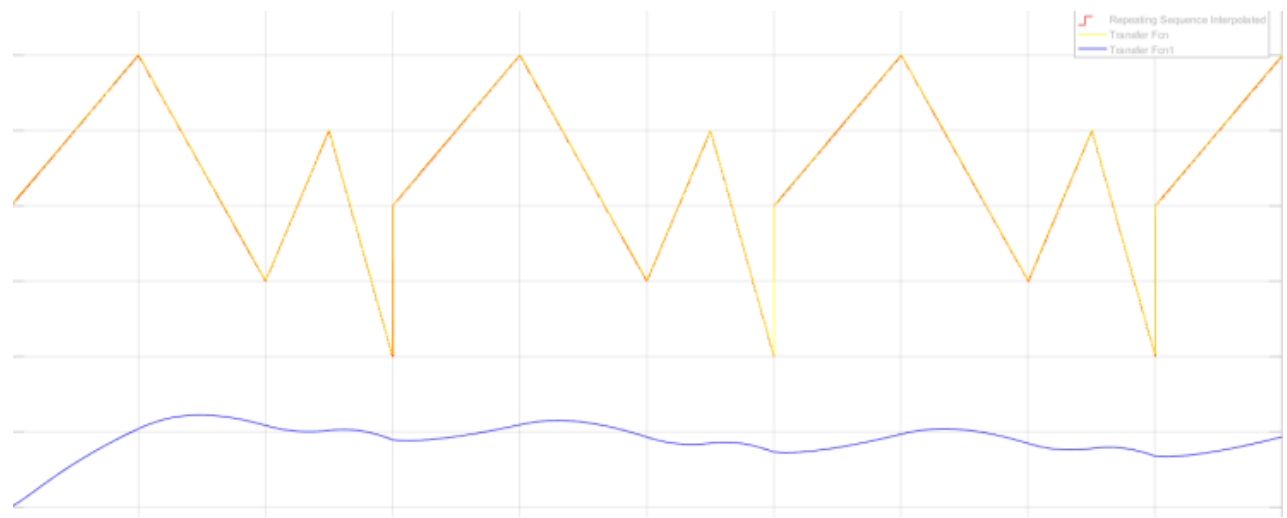

Figure 4a: PD response of 4th order TF

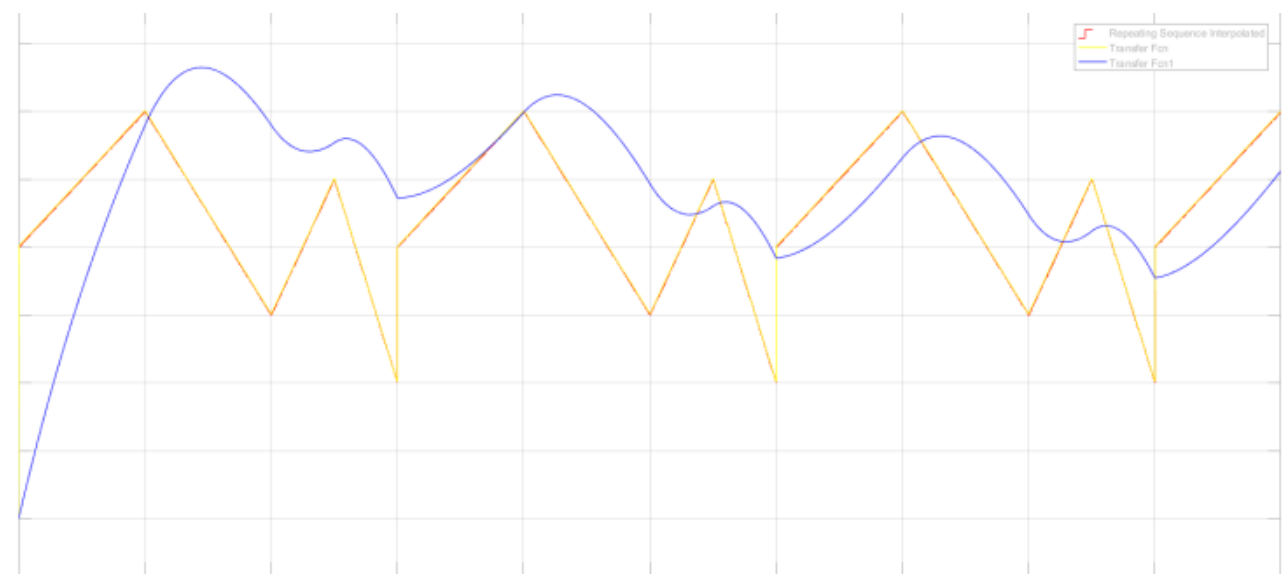

Figure 4b: PD response of 2nd order TF

\section{Conclusion}

Thus, PD controller provides an accurate medium to analyze the behavior under specific environmental constraints. Behavior analysis is done using Simulink considering both second order and fourth order transfer function covering all 6 Degrees of Freedom. Angles computed between different waypoints at different time intervals are given as an input to the ship. The output heading angle is following input heading angle with slight variations of about $0.03 \%$ due to various sea disturbances. Simulation results with no settling time showed PD controller as the best suited option for trajectory control of unmanned surface water vehicle.

\section{References}

[1] Dr. S.A.Hariprasad, Pampapathi, Vijay Singh, Dr. Krishna, Sharath. K, T.D. Shashikala.(2012). "A DESIGN APPROACH TO RUDDER CONTROLLER." International Journal of Computer Science and technology, 3(3), 23-29. 
Recent Advances in Communication, Electronics \& Electrical Engineering

[2] Dr. S.A.Hariprasad, Dr.M. Krishna, Cdr.Vijay Singh, Pampapathi, Sanjay.N, Arunlal.K.S.(2013). "PERFORMANCE ANALYSIS OF SHIP TRACKING USING PID/PREDICTIVE CONTROLLER.” International Journal of Science Engineering and Advance Technology, 1(1), $1-3$.

[3] Ching-Yaw Tzeng, Ju-Fen Chen. (1999). "FUNDAMENTAL PROPERTIES OF LINEAR SHIP STERRING DYNAMIC MODELS.” Journal of Marine Science and Technology, 7(2), 79-88.

[4] Thor I. Fossen. "GUIDANCE AND CONTROL OF OCEAN VEHICLES." John Wiley and Sons LTD (1994).

[5] Ke Feng, Ning Wang, Dan Liu, Meng Joo Er.(2016).“ADAPTIVE FUZZY TRAJECTORY CONTROL OF UNMANNED SURFACE VEHICLES WITH UNKNOWN DYNAMICS." International Conference on Informative and Cybernetics for Computational Social Systems (ICCSS), 1-6.

[6] Yan-Cheng Liu, Si-Yuan Liu, Ning Wang. (2016). "FULLY-TUNED NEURAL NETWORK BASED ROBUST ADAPTIVE TRACKING CONTROL OF UNMANNED UNDERWATER VEHIVLE WITH THRUSTER DYNAMICS.” International Journal of Neurocomputing, 2-28. http://dx.doi.org/10.1016/j.neucom.2016.02.042.

[7] Ning Wang, Jing-chao Sun, Meng Joo, Yan-Cheng Liu. (2015). "A NOVEL LEARNING CONTROL FRAMEWORK OF UNMANNED SURFACE VEHICLES.” IEEE Transcation on Cybernetics, 1-12. 10.1109/TCYB.2015.2423635

[8] Chang-Zhong Pan, Xu-Zhi Lai, Simon X. Yang, Min Wu. (2013). “AN EFFICIENT NEURAL NETWORK APPROACH TO TRACKING CONTROL OF AN AUTONOMOUS SURFACE VEHICLE WITH UNKNOWN DYNAMICS.” Expert System with Applications, 40, 1629-1635.

[9] A.Pedro Aguiar, Joao P. hespanha.(2007)."TRAJECTORY-TRACKING AND PATHFOLLOWING OF UNDERACTUATED AUTONOMOUS VEHICLES WITH PARAMETRIC MODELING UNCERTAINITY.” IEEE Transaction on Automatic Control, 52(8), 1362-1379. 10.1109/TAC.2007.902731.

[10] Khac Duc Do. (2011). "GLOBAL ROBUST AND ADAPTIVE OUTPUT FEEDBACK DYNAMIC POSITIONING OF SURFACE SHIPS.” J. Marine Sci. Appl. 10, 325-332. 10.1007/s11804-0111076-Z

\footnotetext{
*Corresponding author.

E-mail address: adinatha@rvce.edu.in
} 